M. E. Patti - G. McMahon - E. C. Mun - A. Bitton •

J. J. Holst · J. Goldsmith · D. W. Hanto $\cdot$ M. Callery $\cdot$

R. Arky $\cdot$ V. Nose $\cdot$ S. Bonner-Weir $\cdot$ A. B. Goldfine

\title{
Severe hypoglycaemia post-gastric bypass requiring partial pancreatectomy: evidence for inappropriate insulin secretion and pancreatic islet hyperplasia
}

Received: 6 April 2005 / Accepted: 13 June 2005 / Published online: 30 September 2005

(C) Springer-Verlag 2005

Abstract Aims/hypothesis: Postprandial hypoglycaemia following gastric bypass for obesity is considered a late manifestation of the dumping syndrome and can usually be

M. E. Patti $(\bowtie) \cdot$ S. Bonner-Weir · A. B. Goldfine

Research Division, Joslin Diabetes Center,

1 Joslin Place,

Boston, MA 02215, USA

e-mail: mary.elizabeth.patti@joslin.harvard.edu

Tel.: +1-617-7351966

Fax: +1-617-7351970

M. E. Patti · E. C. Mun · A. B. Goldfine

Department of Medicine,

Beth Israel Deaconess Medical Center,

Boston, MA, USA

M. E. Patti · E. C. Mun · J. Goldsmith · D. W. Hanto ·

M. Callery · R. Arky · V. Nose - S. Bonner-Weir · A. B. Goldfine

Harvard Medical School,

Boston, MA, USA

G. McMahon · A. Bitton · R. Arky

Department of Medicine,

Brigham and Women's Hospital,

Boston, MA, USA

E. C. Mun · D. W. Hanto · M. Callery

Department of Surgery,

Beth Israel Deaconess Medical Center,

Boston, MA, USA

\section{J. J. Holst}

Department of Medical Physiology,

University of Copenhagen,

Copenhagen, Denmark

J. Goldsmith

Department of Pathology,

Beth Israel Deaconess Medical Center,

Boston, MA, USA

V. Nose

Department of Pathology,

Brigham and Women's Hospital,

Boston, MA, USA managed with dietary modification. We investigated three patients with severe postprandial hypoglycaemia and hyperinsulinaemia unresponsive to diet, octreotide and diazoxide with the aim of elucidating the pathological mechanisms involved. Methods: Glucose, insulin, and C-peptide were measured in the fasting and postprandial state, and insulin secretion was assessed following selective intra-arterial calcium injection. Pancreas histopathology was assessed in all three patients. Results: All three patients had evidence of severe postprandial hyperinsulinaemia and hypoglycaemia. In one patient, reversal of gastric bypass was ineffective in reversing hypoglycaemia. All three patients ultimately required partial pancreatectomy for control of neuroglycopenia; pancreas pathology of all patients revealed diffuse islet hyperplasia and expansion of beta cell mass. Conclusions/interpretation: These findings suggest that gastric bypass-induced weight loss may unmask an underlying beta cell defect or contribute to pathological islet hyperplasia, perhaps via glucagon-like peptide 1-mediated pathways.

Keywords Gastric bypass · GLP1 · Glucagon-like peptide 1 - Hypoglycaemia · Incretin - Insulin · Obesity

Abbreviations CT: computerised tomography - GLP1: glucagon-like peptide $1 \cdot$ MEN-I: multiple endocrine neoplasia I · PTH: parathyroid hormone

\section{Introduction}

Postprandial hypoglycaemia following gastric bypass is considered to be a late manifestation of the dumping syndrome and can usually be managed with dietary modification. We report on three patients with severe postprandial hypoglycaemia and hyperinsulinaemia unresponsive to medical intervention, who ultimately required partial pancreatectomy for control of neuroglycopenia. Pathology revealed diffuse islet hyperplasia and expansion of beta cell mass. 


\section{Patient 1}

History and examination A 27-year-old female with obesity dating to childhood underwent a vertical banded gastroplasty for severe obesity (BMI $39 \mathrm{~kg} / \mathrm{m}^{2}$ ). While there was no personal or family history of diabetes, the patient's mother and sister also had severe obesity and underwent bariatric surgery. Despite a weight loss of about $45 \mathrm{~kg}$ in the first year postoperatively, she required surgical re-exploration 1 year later for mesh erosion, at which time gastroplasty was converted to a roux-en-Y gastric bypass. Within the next 12 months, when her BMI had stabilised at $24 \mathrm{~kg} / \mathrm{m}^{2}$, she developed progressive postprandial hypoglycaemia, which was unresponsive to dietary intervention, phenytoin, beta-blockers, acarbose or diazoxide. Despite take-down of the gastric bypass 3 years following the initial procedure and regain of nearly all of weight lost, episodic hypoglycaemia increased in severity and frequency, with some episodes not clearly linked to food intake. Documented glucose levels as low as $1.1 \mathrm{mmol} / 1$ were associated with loss of consciousness and a motor vehicle accident.

Investigation Laboratory evaluation during one symptomatic episode revealed glucose $2.2 \mathrm{mmol} / \mathrm{l}$, with concurrent insulin $72 \mathrm{pmol} / \mathrm{l}$ and $\mathrm{C}$-peptide $0.87 \mathrm{nmol} / \mathrm{l}$ (reference ranges: fasting insulin $<21 \mathrm{pmol} / 1$; C-peptide $0.37-1.07 \mathrm{nmol} / 1)$. Tests for anti-insulin antibodies and a sulfonylurea screen were negative. Abdominal computerised tomography (CT), magnetic resonance imaging and an octreotide scan revealed no pancreatic lesions. To localise the site of inappropriate insulin secretion, selective arterial injection of calcium was performed [1]. Insulin levels were similar prior to and following selective intra-arterial calcium injection (splenic artery $51-85 \mathrm{pmol} / \mathrm{l}$, gastroduodenal artery $232-276 \mathrm{pmol} / 1$ and superior mesenteric artery $286-304 \mathrm{pmol} / \mathrm{l})$.

Seven years after the initial vertical banded gastroplasty, the patient underwent exploratory laparotomy for her re-
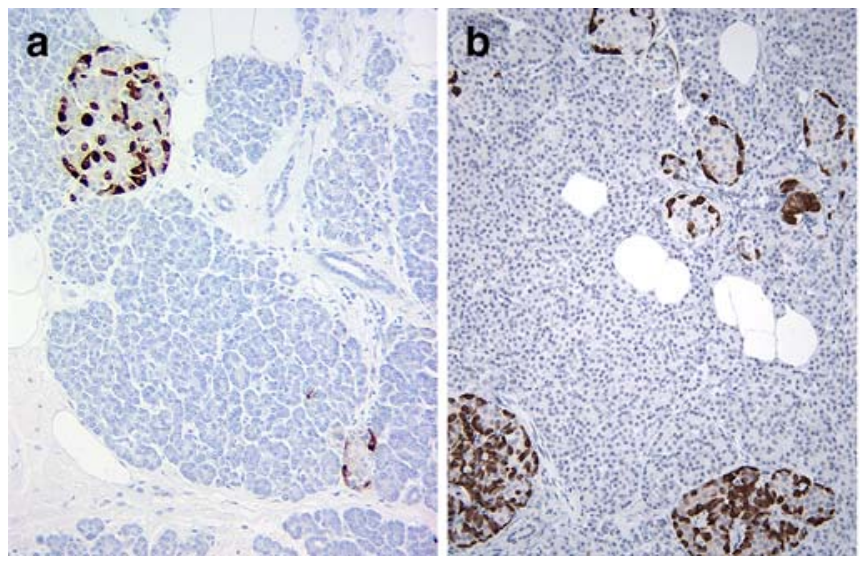

Fig. 1 a Normal endocrine and exocrine pancreas visualised with immunohistochemical staining for glucagon (black). b Patient 1. Hyperplasia of islets seen by immunohistochemical staining for glucagon (black). c Patient 2. Hyperplasia of islets seen by immunohistochemical staining for glucagon (black). Individual islets have a normal organisation for humans but are in aberrant current hyperinsulinaemic hypoglycaemia. Neither visual/ palpation inspection nor intra-operative ultrasound revealed an insulinoma; an $80 \%$ distal pancreatectomy was performed. Pathological examination revealed variably sized islets, proliferation of single islet cells, and diffuse nesidioblastosis with no solitary lesion identified (Fig. 1b). Postoperatively, she continued to have three to five episodes of hypoglycaemia daily, often requiring glucagon. Given the persistence and frequency of symptoms unresponsive to dietary and pharmacological intervention, she underwent total pancreatectomy (Whipple procedure) 2 years later. Pathology was again consistent with diffuse islet hyperplasia and nesidioblastosis.

\section{Patient 2}

History and examination A 45-year-old male underwent roux-en-Y gastric bypass and distal gastrectomy for severe obesity dating to adolescence (BMI $42 \mathrm{~kg} / \mathrm{m}^{2}$ ). His postoperative course was complicated by a duodenal stump leak, biliary stricture and short-gut syndrome from a long Roux limb, ultimately requiring a choledochojejunostomy and bypass revision. He had no personal or family history of diabetes or hypoglycaemia.

Between 9 and 12 months postoperatively, when his BMI was stable at $22 \mathrm{~kg} / \mathrm{m}^{2}$, he developed adrenergic and neuroglycopenic symptoms of hypoglycaemia, including palpitations, sweating, profound fatigue, confusion, disorientation and morning headaches. Evaluations of hepatic, renal, adrenal, pituitary and IGF-1 axis functions were normal. Capillary and continuous subcutaneous glucose monitoring revealed a correlation between symptoms and declining or frankly low glucose $(<2.8 \mathrm{mmol} / \mathrm{l})$. Following a mixed meal challenge (Glucerna, $29 \mathrm{~g}$ carbohydrate; Abbott Laboratories, Abbott Park, IL, USA), insulin peaked at $1808 \mathrm{pmol} / 1$ (30 min); minimum glucose was $2.4 \mathrm{mmol} / 1$ (60 $\mathrm{min})$. Initial clinical impression was hypoglycaemia associated with
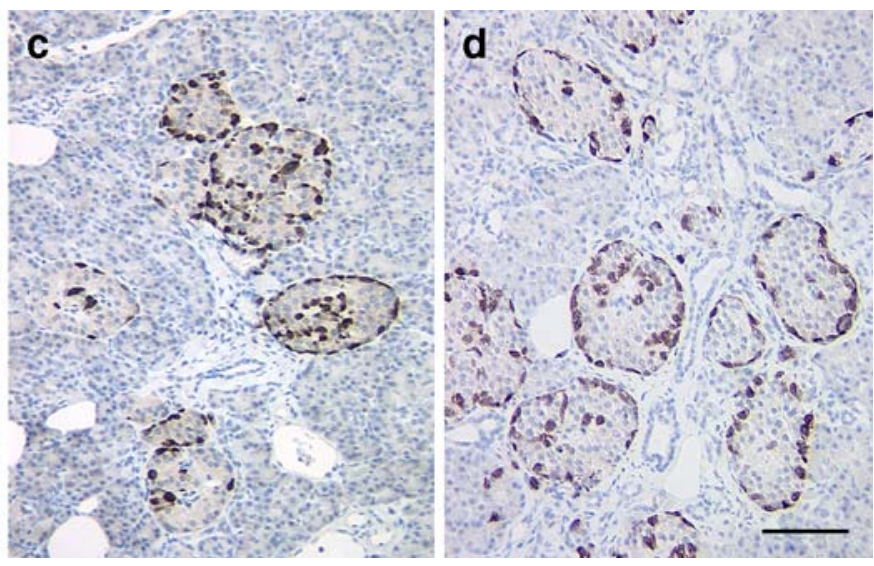

clusters surrounding ductal profiles. Islets (centre) are seen in close proximity to ductules that have glucagon-positive cells in their epithelium. d Patient 3. Hyperplasia of islets seen by immunohistochemical staining for glucagon (black). Magnification bar (panel d) represents $100 \mu \mathrm{m}$ 
dumping syndrome, aggravated by chronic undernutrition. Despite avoidance of simple carbohydrates, symptoms increased in severity and frequency, with hypoglycaemia $(1.4 \mathrm{mmol} / \mathrm{l})$ resulting in a motor vehicle accident.

Investigation Protein challenge $(1 \mathrm{~g} / \mathrm{kg}$ Promod; Abbott Laboratories) did not provoke hypoglycaemia, hyperinsulinaemia or hyperammonaemia. An inpatient fast $(72 \mathrm{~h})$ was negative (glucose $3.4 \mathrm{mmol}$, insulin $<14 \mathrm{pmol} / \mathrm{l}, \beta$ hydroxybutyrate $4.4 \mathrm{mmol} / \mathrm{l}$ at $72 \mathrm{~h}$ ). Clinical assessment was that of alimentary hypoglycaemia. The patient was discharged on preprandial octreotide and supplementary jejunostomy feedings with a custom mixture of cornstarch, Promod and Benecalorie (Novartis Medical Nutrition, Basel, Switzerland). Tube feedings were discontinued after 3 months due to insertion site irritation.

Despite dietary modification, cornstarch supplements, octreotide and weight maintenance, neuroglycopenia increased in frequency and severity. Neither diazoxide $(150 \mathrm{mg}$ three times per day) nor nifedipine attenuated hypoglycaemia.

Random outpatient glucose level was $2.4 \mathrm{mmol} / \mathrm{l}$, with concomitant insulin $71 \mathrm{pmol} / \mathrm{l}$, C-peptide $1.50 \mathrm{nmol} / \mathrm{l}$, proinsulin $45 \mathrm{pmol} / \mathrm{l}$, glucagon $130 \mathrm{ng} / \mathrm{l}, \beta$-hydroxybutyrate $0.2 \mathrm{mmol} / \mathrm{l}$, and negative sulfonylurea and repaglinide screens. Glucagon response was present. Formal mixed meal challenge (Ensure, $40 \mathrm{~g}$ carbohydrate; Abbott Laboratories) demonstrated increased insulin and C-peptide within $30 \mathrm{~min}$ (peak $2,705 \mathrm{pmol} / 1$ and $6.23 \mathrm{nmol} / 1$, respectively), with glucose nadir of $3.3 \mathrm{mmol} / \mathrm{l}$ at $2 \mathrm{~h}$. Abdominal CT with spiral pancreatic cuts showed no lesions. To localise the site of inappropriate insulin secretion, selective arterial injection of calcium was again performed [1]. Insulin levels in the hepatic vein prior to and following calcium injection were as follows: splenic artery 45-194 pmol/1, gastroduodenal artery 51-108 pmol/1, and superior mesenteric artery $27-21 \mathrm{pmol} / \mathrm{l}$. These data suggested that insulin secretion was localised to the mid-pancreas and tail. Multiple endocrine neoplasia I (MEN-I) mutation analysis was negative. Serum vasoactive intestinal polypeptide, gastrin, parathyroid hormone $(\mathrm{PTH})$, chromogranin A, serotonin and urinary hydroxyindoleacetic acid (HIAA) levels were normal. Anti-insulin antibodies were absent.

Because of the increasing frequency and severity of neuroglycopenia, surgical options were considered. Since reversal of gastric bypass and gastrectomy was not possible due to surgical anatomy, a distal pancreatectomy, just to the right of the superior mesenteric vein, was performed. Neither intraoperative ultrasound nor visual/pathological inspection demonstrated an insulinoma. Histopathology revealed diffuse islet hyperplasia with small islets adjacent to, and budding from, ducts (Fig. 1c). Cells positively stained for insulin, glucagon and chromogranin were abundant in ducts. Ki-67 staining was prominent in ducts. Beta cell relative volume was $3.3 \%$, a value higher than the $2.6 \pm 0.4 \%$ seen in pancreas sections from obese non-diabetic patients $[2,3]$. There was no evidence of chronic pancreatitis. The patient's postoperative course was uncomplicated and the hypoglycaemia was resolved for several months. He has recently had some recurrence of hypoglycaemia, albeit decreased in frequency and severity compared with preoperative symptoms.

Peripheral blood DNA analysis revealed no mutations in the genes encoding sulphonylurea receptor 1 (SUR1, $A B C C 8$ ), inwardly rectifying potassium channel (Kir6.2, KCNJ11) or glucokinase associated with congenital hyperinsulinaemia (Athena Diagnostics, Worcester, MA, USA). Duplicate analysis of prepancreatectomy blood samples demonstrated striking elevations in glucagon-like peptide 1 (GLP1; C-terminal assay), which reached a maximum of $328 \mathrm{pmol} / \mathrm{l}$ at 30 min after a mixed meal (normal values for healthy controls $<30 \mathrm{pmol} / 1$ fasting, postprandial peak $<60 \mathrm{pmol} / 1$, $<100 \mathrm{pmol} / \mathrm{l}$ in dumping syndrome patients) $[4,5]$.

\section{Patient 3}

History and examination A 66-year-old female underwent a roux-en-Y gastric bypass for severe obesity dating back to adolescence (BMI $48 \mathrm{~kg} / \mathrm{m}^{2}$ ). Two years later, when her BMI had stabilised at $35 \mathrm{~kg} / \mathrm{m}^{2}$, the patient noted the onset of intermittent diaphoresis, palpitations and confusion, usually occurring $1.5-3 \mathrm{~h}$ postprandially. Capillary glucose was as low as $1.4 \mathrm{mmol} / \mathrm{l}$ with home monitoring, while laboratory evaluation at the time of spontaneous symptoms demonstrated glucose $3.2 \mathrm{mmol} / 1$, insulin $79 \mathrm{pmol} / \mathrm{l}$ and C-peptide $0.97 \mathrm{nmol} / \mathrm{l}$. There was no fasting hypoglycaemia.

Formal mixed meal challenge (Ensure, $40 \mathrm{~g}$ carbohydrate; Abbott Laboratories) demonstrated increased insulin and C-peptide within $30 \mathrm{~min}$ (peak 2,253 pmol/1 and $6.96 \mathrm{nmol} / 1$, respectively), with a glucose nadir of $3.1 \mathrm{mmol} / 1$ at $2 \mathrm{~h}$. GLP1 levels peaked at $>300 \mathrm{pmol} / \mathrm{l}$ (above upper limit of assay). Abdominal CT with spiral pancreatic cuts showed no lesions. To localise the site of inappropriate insulin secretion, selective arterial injection of calcium was performed [1]. Insulin levels in the hepatic vein prior to and following calcium injection were as follows: splenic artery 29-144 pmol/1, gastroduodenal artery $29-172 \mathrm{pmol} / \mathrm{l}$ and superior mesenteric artery $29-50 \mathrm{pmol} / \mathrm{l}$. These data suggested that insulin secretion was diffuse throughout the pancreas. Serum gastrin, PTH and chromogranin A levels were normal. DNA analysis was negative for mutations in the genes encoding SUR1, Kir6.2 and glucokinase.

Despite strict dietary intervention, octreotide therapy, diltiazem and a trial of diazoxide (discontinued due to lack of efficacy and side effects), neuroglycopenia increased in frequency (up to three episodes per day) and magnitude, with falls, loss of consciousness and witnessed seizure-like activity. A subtotal (85\%) pancreatectomy was performed. Intra-operative ultrasound and detailed visual/pathological inspection was negative for insulinoma. Histopathology revealed diffuse islet hyperplasia (Fig. 1d). There was no evidence of chronic pancreatitis. Postoperative course has been uncomplicated, with no hypoglycaemia to date. 


\section{Conclusions}

In this paper we describe three post-gastric bypass patients with severe postprandial hypoglycaemia because of inappropriate insulin secretion and major islet hyperplasia. We have observed similar severe, postprandial hyperinsulinaemia and hypoglycaemia in other post-gastric bypass patients requiring octreotide for control, who have not undergone pancreatectomy. Potential mechanisms include: (1) profound dumping syndrome; (2) beta cell hyperfunction; (3) improved insulin sensitivity following weight loss unmasking an underlying hyperinsulinaemia syndrome; (4) lack of regression of increased functional beta cell mass that developed during prior obesity; and (5) GLP1mediated active expansion of beta cell mass.

Hypoglycaemia post-gastric bypass can be a late manifestation of the dumping syndrome, and may be associated with a constellation of symptoms, including palpitations, lightheadedness, abdominal cramping and diarrhoea. While initial evaluation suggested that our patients' postprandial hypoglycaemia was most likely related to dumping, the severity of hypoglycaemia with neuroglycopenia, lack of response to dietary and medical therapy, and progression over time suggested a more severe dysregulation of insulin secretion and the presence of additional pathology.

Uncommonly, postprandial hypoglycaemia may be linked to beta cell hyperfunction, including insulinoma, non-insulinoma pancreatogenous hypoglycaemia and familial hyperinsulinaemia. Insulinoma has been reported in a postgastric bypass patient with both fasting and postprandial hypoglycaemia [6]. While isolated postprandial hypoglycaemia is a rare presentation of insulinoma, detailed pathological examination demonstrated no insulinoma in our patients.

Noninsulinoma pancreatogenous hypoglycaemia is a rare syndrome described by Service et al. [7], characterised by isolated postprandial hypoglycaemia, hyperinsulinaemia and negative 72-h fast. Partial pancreatectomy, guided by insulin secretory gradients, was required for ten adult patients. Despite the diffuse nature of islet hypertrophy and nesidioblastosis on pathology, symptoms were either resolved or palliated by surgery [8]. To date, a genetic basis for this syndrome has not been identified.

Familial hyperinsulinism may be identified in adult family members only after a neonate is diagnosed with persistent hyperinsulinemic hypoglycaemia of infancy. Mutations in SUR1, Kir6.2, glucokinase, glutamate dehydrogenase and short-chain 3-hydroxyacyl CoA dehydrogenase account for the majority of cases. SUR1 mutations, usually inherited in an autosomal recessive pattern, tend to be more severe and often require pancreatectomy. Autosomal dominant forms, which may be milder [9], have been linked to the gene encoding glucokinase $(G C K)$ in some families [10]. Additional familial forms are characterised by exercise-induced insulin secretion, but the mutation(s) responsible have not been identified [11].
It is also possible that the weight loss induced by bariatric surgery and the associated improvement in insulin sensitivity unmasked an underlying familial hyperinsulinism syndrome or primary insulin hypersecretion [12], either of which may have also contributed to the development of obesity. However, there were no signs or symptoms of hypoglycaemia in the patients prior to bariatric surgery. While weight loss and improved insulin sensitivity would be expected to lead to normalisation of increased beta cell function and/or mass in previously obese subjects [12], it is also possible that failure of regression played a pathological role in our patients. However, the Ki-67 staining suggests ongoing expansion of beta cell mass.

We believe that excessive secretion of GLP1 played a pivotal role in post-gastric bypass hypoglycaemia in our second and third patients, and perhaps in the first. Distal intestinal delivery of nutrients post-gastrectomy stimulates GLP1 and/or gastric inhibitory polypeptide secretion, increasing insulin secretion and contributing to alimentary hypoglycaemia [5]. Furthermore, GLP1 can also produce islet hyperplasia in rodents [13]. However, abnormal pancreatic histology has not been reported post-gastrectomy in humans to date.

In summary, while enhanced insulin secretion post-gastric bypass is a component of the dumping syndrome, the severity of hypoglycaemia/hyperinsulinaemia in some patients suggests a newly recognised pathological syndrome. Potential mechanisms in our patients include: (1) improved insulin sensitivity post-weight loss unmasking an underlying familial hyperinsulinaemia syndrome; (2) lack of regression of increased beta cell mass that developed with prior obesity; or (3) GLP1-mediated active expansion of beta cell mass. In our patients, active expansion of beta cell mass via islet neogenesis or replication as the major mechanism contributing to hyperinsulinaemia is suggested by the postoperative lag time to severe hypoglycaemia and the active proliferation observed in pathological specimens.

Further investigation will be critical for the elucidation of the mechanisms of beta cell expansion in the adult human. More importantly, given the increasing prevalence of gastric bypass, clinicians may wish to consider that severe hypoglycaemia in post-gastric bypass patients unresponsive to nutritional modification may indicate an underlying disorder of beta cell function.

\section{Note added in proof}

Six similar post-bypass patients, with severe hypoglycaemia requiring pancreatectomy, were recently reported by Service et al.

Service GJ, Thompson GB, Service FJ et al (2005) Hyperinsulinemic hypoglycemia with nesidioblastosis after gastric-bypass surgery. $N$ Engl J Med 353:249-254.

Acknowledgements This work was supported by NIH grants DK062948 (M. E. Patti), DK44523 (S. Bonner-Weir), and DK02795 (A. B. Goldfine), the Julie Henry Fund, Beth Israel Deaconess Medical Center (D. W. Hanto) and the General Clinical Research Center (M01-RR01032). We appreciate helpful discussion with J. Service. 


\section{References}

1. Doppman JL, Chang R, Fraker DL et al (1995) Localization of insulinomas to regions of the pancreas by intra-arterial stimulation with calcium. Ann Intern Med 123:269-273

2. Yoon KH, Ko SH, Cho JH et al (2003) Selective beta-cell loss and alpha-cell expansion in patients with type 2 diabetes mellitus in Korea. J Clin Endocrinol Metab 88:2300-2308

3. Butler AE, Janson J, Bonner-Weir S, Ritzel R, Rizza RA, Butler PC (2003) Beta-cell deficit and increased beta-cell apoptosis in humans with type 2 diabetes. Diabetes 52:102-110

4. Vilsboll T, Krarup T, Sonne J et al (2003) Incretin secretion in relation to meal size and body weight in healthy subjects and people with type 1 and type 2 diabetes mellitus. J Clin Endocrinol Metab 88:2706-2713

5. Gebhard B, Holst JJ, Biegelmayer C, Miholic J (2001) Postprandial GLP-1, norepinephrine, and reactive hypoglycemia in dumping syndrome. Dig Dis Sci 46:1915-1923

6. Zagury L, Moreira RO, Guedes EP, Coutinho WF, Appolinario JC (2004) Insulinoma misdiagnosed as dumping syndrome after bariatric surgery. Obes Surg 14:120-123

7. Service FJ, Natt N, Thompson GB et al (1999) Noninsulinoma pancreatogenous hypoglycemia: a novel syndrome of hyperinsulinemic hypoglycemia in adults independent of mutations in Kir6.2 and SUR1 genes. J Clin Endocrinol Metab 84: $1582-1589$
8. Thompson GB, Service FJ, Andrews JC et al (2000) Noninsulinoma pancreatogenous hypoglycemia syndrome: an update in 10 surgically treated patients. Surgery 128:937-944

9. Thornton PS, Satin-Smith MS, Herold K et al (1998) Familial hyperinsulinism with apparent autosomal dominant inheritance: clinical and genetic differences from the autosomal recessive variant. J Pediatr 132:9-14

10. Cuesta-Munoz AL, Huopio H, Otonkoski T et al (2004) Severe persistent hyperinsulinemic hypoglycemia due to a de novo glucokinase mutation. Diabetes 53:2164-2168

11. Otonkoski T, Kaminen N, Ustinov J et al (2003) Physical exercise-induced hyperinsulinemic hypoglycemia is an autosomal-dominant trait characterized by abnormal pyruvate-induced insulin release. Diabetes 52:199-204

12. Ferrannini E, Camastra S, Gastaldelli A et al (2004) Beta-cell function in obesity: effects of weight loss. Diabetes 53(Suppl 3): S26-S33

13. Drucker DJ (2003) Enhancing incretin action for the treatment of type 2 diabetes. Diabetes Care 26:2929-2940 